\title{
New Development for Safer Endoscopic Submucosal Dissection using the Carbon Dioxide $\left(\mathrm{CO}_{2}\right)$ Laser (Secondary publication)
}

\author{
Yoshinori Morita ${ }^{1,2}$, Ryusuke Ariyoshi ${ }^{2}$, Norihiro Honda ${ }^{3,4}$, \\ Hisanao Hazama ${ }^{3}$, Yuzo Kodama ${ }^{2}$, Kunio Awazu 3, 5, 6 \\ 1: Department of Gastroenterology, Kobe University International Clinical Cancer Research Center \\ 2: Department of Gastroenterology, Kobe University Graduate School of Medicine \\ 3: Division of Sustainable Energy and Environmental Engineering, \\ Graduate School of Engineering, Osaka University \\ 4: Institute of Innovative Research, Osaka University \\ 5: Graduate School of Frontier Biosciences, Osaka University \\ 6: Global Center for Medical Engineering and Informatics, Osaka University
}

\begin{abstract}
Endoscopic Submucosal Dissection (ESD) has been increasingly accepted as a minimally invasive treatment for the patients with early gastrointestinal cancers. However, reliable ESD technique demands high maneuverability, and the success of the operation is dependent on each operator's skill. We have developed a novel laser endoscopic system for ESD to overcome such technique-related difficulties. Compared to conventional ESD using electrosurgical knives, an ESD method using the $\mathrm{CO}_{2}$ laser offers the advantages of less risk of perforation and the prevention of extensive thermal damage, because the $\mathrm{CO}_{2}$ laser is strongly absorbed by water. Furthermore, due to the non-contact technique associated with the $\mathrm{CO}_{2}$ laser and adequate visualization of the treatment area, the laser system may provide every endoscopist with more precise and safer treatment.
\end{abstract}

Key words: Endoscopic submucosal dissection $(\mathrm{ESD}) \cdot \mathrm{CO}_{2}$ laser, hollow optical fiber • perforation $\cdot$ bleeding

\section{Introduction}

The development of the first carbon dioxide $\left(\mathrm{CO}_{2}\right)$ laser was firstly reported by C.K.N Patel in 1964. The $\mathrm{CO}_{2}$ laser has the following characteristics: the wavelength is 10.6 $\mu \mathrm{m}$, which is highly absorbed by water and the thermal coagulation depth in the target tissue caused by the incident $\mathrm{CO}_{2}$ laser energy is shallower than that of the electrosurgical knife. ${ }^{1)}$ These characteristics lead to the development of the so-called 'laser scalpel' which is to this day widely used on soft tissues in the fields of dental/oral surgery, otolaryngologic surgery, neurosurgery and in dermatologic surgery.

On the other hand, in the field of gastroenterologi-

Addressee for Correspondence:

Yoshinori Morita, M.D. \& Ph.D.

7-5-1, Kusunoki-cho, Chuo-ku, Kobe, Hyogo,

650-0017 Japan

TEL: +81-78-382-6305, FAX: +81-78-382-6309

Email: ymorita@med.kobe-u.ac.jp cal surgery, the first study involving laser treatment was reported in 1981 where a high output Neodymium: Yttrium Aluminum Garnet (Nd:YAG) laser was used to ablate an early gastric cancer lesion ${ }^{2}$. Although the deep tissue penetration characteristic of the Nd:YAG laser energy made it highly effective for tissue coagulation and hemostasis, at the same time it was unsuitable for use as a precision laser scalpel for that very same reason. Further developments of the use of laser in the field of gastroenterology comprised the indication of the low output excimer dye laser (EDL) and high tumor affinity photosensitizing agents (photosensitizers: PS), specifically porfimer sodium, known as Photofrin ${ }^{\circledR}$, which used in combination allowed for tumor-specific destruction through a photochemical cascade. Such treatment is referred to as photodynamic therapy (PDT) ${ }^{3)}$ and recent developments in PDT with the use of diode laser energy

Received date: August 10th, 2018

Accepted date: April 20th, 2019

Laser Therapy 28.2: 89-96 
combined with Laserphyrin (talaporfin sodium) as the photosensitizer is considered to be the second generation PDT. However, the indication of PDT in Japan is limited to superficial esophageal and early gastric cancers of inoperable and unsuited for endoscopic resection. PDT is recently most used as a salvage treatment for local recurrences of esophageal squamous cell carcinoma following chemoradiation therapy ${ }^{4,5)}$

In the field of gastroenterology, the role and importance of the endoscopic approach has been becoming vital for the treatment of early gastrointestinal cancers from the standpoint of low invasiveness. Endoscopic mucosal resection (EMR) which was developed in the 1980s is performed by first injecting fluids such as normal saline into the submucosal layer directly underneath the lesion thereby lifting the lesion. A pre-looped metallic snare is introduced to the elevated lesion, constriction of the snare and electrical cauterization follows, and the lesion is resected. This procedure is relatively simple and its use has spread throughout the world. However, when the size of the lesion is greater than $2 \mathrm{~cm}$, larger than the size of the snare, or when the lesion is located at difficult sites, en-bloc resection becomes difficult. However, in the early 2000s, the snare was replaced by electrosurgical knife. The mucosa was cut open to reveal the submucosal layer and direct dissection of the submucosal layer and resection of the lesion was achieved. This newly developed procedure was named endoscopic submucosal dissection (ESD). This procedure made possible the enbloc resection of lesions regardless of the size and location of the lesion and allowed for more detailed pathological evaluations. This made-in Japan innovative procedure is something the Japanese should be proud of and is spreading both domestically and internationally year by year. However, it does have its drawbacks such as requiring the endoscopists to have expertise in the procedure. Crude maneuvering of the endoscope or excessive electrocauterization readily lead to perforations and bleeding, especially when the procedure is being performed on a thin-walled gastrointestinal tract ${ }^{6}$. Presently, to improve the safety of the procedure, submucosal injection with viscous agents such as sodium hyaluronate have been attempted to increase the duration of the elevation time of the lesion, and numerous changes in the size and shape of the electrosurgical knife have been developed but the success of the procedure still relies on the skill and experience of each endoscopist. Recently there is an increase in the prevalence of colorectal cancer, probably in proportion to the increase in a Westernized diet, of not only in Japan but throughout the world, and dissemination of a safer and easier endoscopic procedure is warranted. Such can only be achieved through new developments in techniques and hardware.

\section{Purpose}

To develop a safer and easier gastrointestinal endoscopic treatment, the authors firstly focused on the agent used for the submucosal injection. The main component of any agent would be water which would efficiently absorb laser light in the mid-infrared (mid-IR) wavelength. The authors hypothesized that the injections of agents such as normal saline or sodium hyaluronate into the submucosal layer would act as an absorber of mid-IR laser energy and by replacing the electrosurgical knife with a $\mathrm{CO}_{2}$ laser, the injected agents would act as a buffer, making the procedure less invasive for the muscle propria layer and thus realizing a safer mucosal incision and submucosal dissection. The purpose of this study was to develop a novel $\mathrm{CO}_{2}$ laser device to be used with endoscopes Figure 1).

\section{Materials and methods}

\section{The development of $\mathrm{CO}_{2}$ laser device fit for flex- ible endoscopes.}

When using the Nd:YAG laser, laser light was transmitted through conventional silica fibers passed through the forceps channel of the endoscope. In the case of the $\mathrm{CO}_{2} \mathrm{la}-$ ser, this was not possible as laser energy at the $\mathrm{CO}_{2}$ wavelength was absorbed in the normal fiber, destroying it. $\mathrm{CO}_{2}$ laser energy normally needed to be transmitted by multi-jointed mirrors in an articulated arm. The first and foremost requirement was developing a flexible fiber that

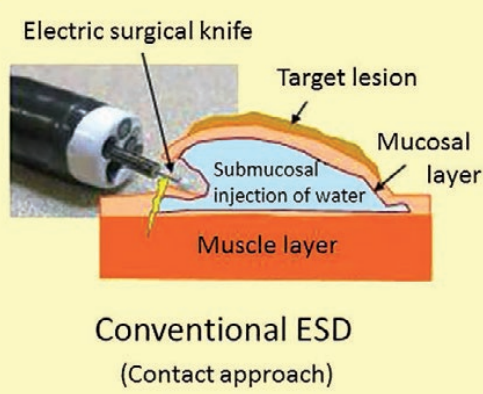

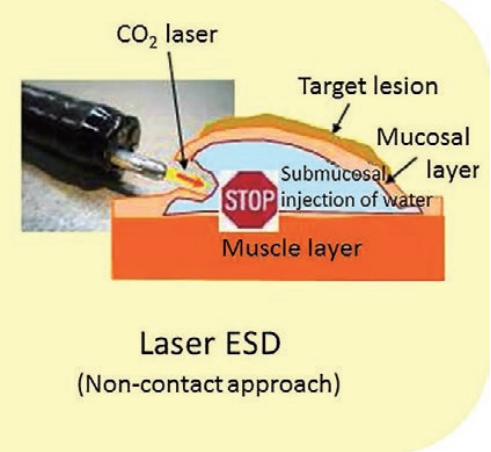

Fig. 1: Comparison between conventional and laser ESD. Compared to conventional ESD using electric surgical knives, ESD using $\mathrm{CO}_{2}$ laser method has an advantage of less risk of perforation and massive thermal damage, because the $\mathrm{CO}_{2}$ laser is strongly absorbed by water in saline or sodium hyaluronate. 
was capable of transmitting $\mathrm{CO}_{2}$ laser energy. This study received technical support from $\mathrm{J}$. Morita MFG Corp. and a novel $\mathrm{CO}_{2}$ laser device was developed where a hollow optical fiber with inner/outer diameters of 0.53/0.66 mm and a length of $2.6 \mathrm{~m}$ was used to transmit the laser light. During actual endoscopic procedures, flexion of the tip of the endoscope may be as much as $210^{\circ}$, but by using a small diameter hollow optical fiber, damage to the fiber due to over-flexion can be avoided and the transmission of $\mathrm{CO}_{2}$ laser energy was made possible. Since $\mathrm{CO}_{2}$ laser light was invisible to the human eye, an aiming beam from a laser in the visible spectrum was required to show the actual incident point of the $\mathrm{CO}_{2}$ laser. This fiber is also capable of simultaneously transmitting visible laser light at the wavelength $642 \mathrm{~nm}$. A small volume of air was pumped through the hollow optical fiber to avoid any clogging within the fiber and water was circulated around the exterior of the fiber to avoid any thermal

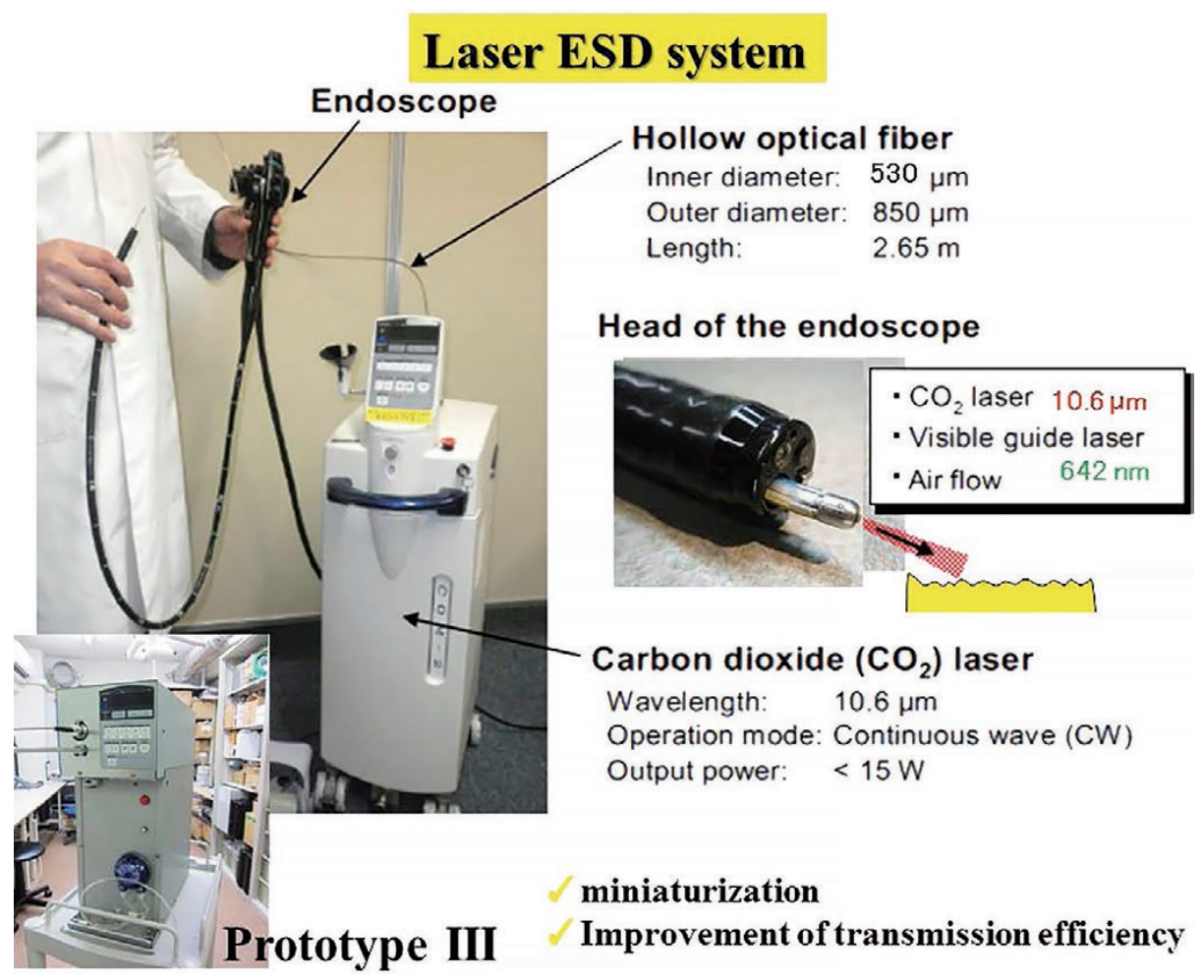

Fig. 2: Laser ESD system. The inner diameter of the fiber was $530 \mu \mathrm{m}$ and the fiber can simultaneously transmit a visible laser as a guide laser. Prototype III was developed for the purpose of miniaturization and improvement of transmission efficiency.

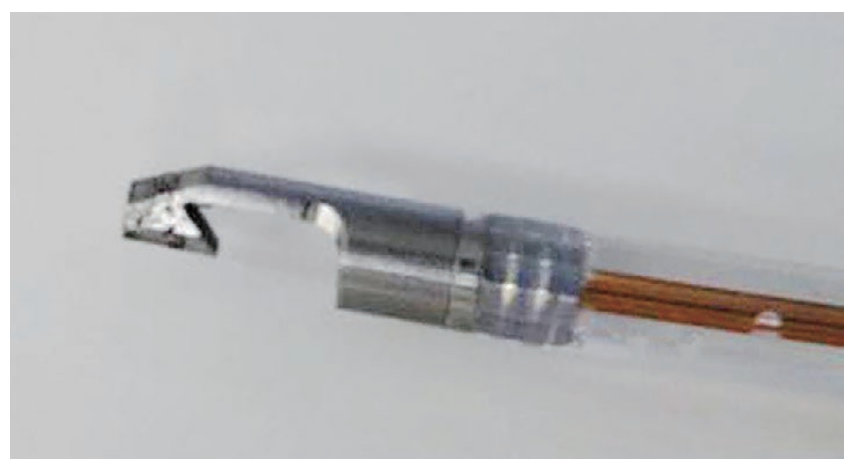

Fig. 3: "Hot tip" device. Vessel coagulation is possible by laser irradiation to the tip of the device and submucosal dissection is possible by using hooking part.

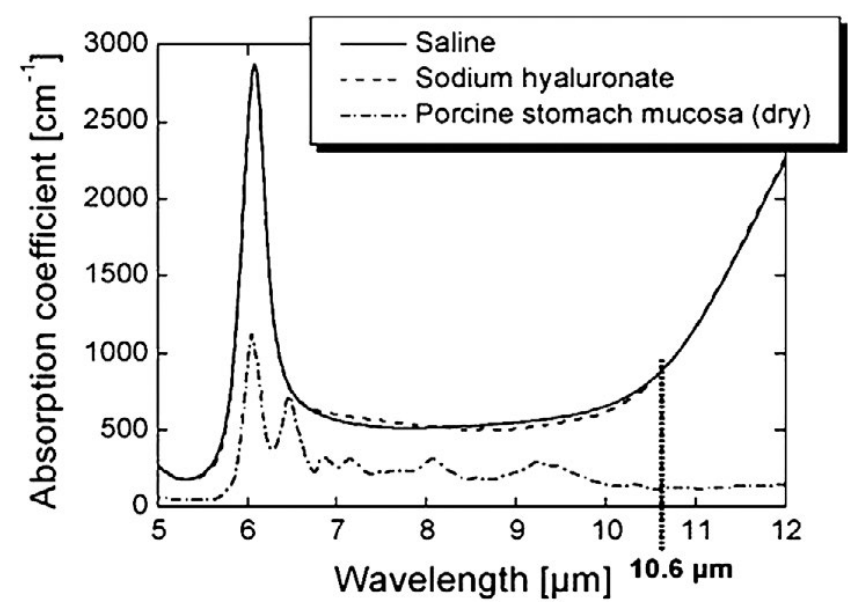

Fig. 4: The infrared absorption spectra of saline, sodium hyaluronate, and porcine stomach mucosa. The spectra of saline and sodium hyaluronate were similar. The absorption coefficients of these solutions were higher than that of the lamina propria at a wavelength of $10.6 \mu \mathrm{m}$. 
damage to the fiber caused by escaping laser energy. The $\mathrm{CO}_{2}$ laser hardware used was a commercially available unit, where modifications were made to miniaturize the unit and improve transmission efficiency (Figure 2).

To deal with intra-procedural hemorrhage and blood vessel sealing, a novel hot-tip type hemostat was also developed. Laser irradiation of the tip of the device heats the tip enabling vessel coagulation. The hooked part of the device allows the grasping of tissue and can be used directly for the dissection (Figure 3)

\section{In vitro experiments}

\section{Relevant absorption spectra of the $\mathrm{CO}_{2}$ laser}

The infrared absorption spectra of saline, sodium hyaluronate and porcine stomach mucosa measured with a micro-Fourier transformation infrared spectrophotometer (FT520, Horiba, Ltd.) was shown in Figure 4. At the wavelength of $10.6 \mu \mathrm{m}$, the wavelength of the $\mathrm{CO}_{2}$ laser, the absorption coefficients of the injection agents were 8 times higher than that of the stomach mucosa, which meant that, as Figure $\mathbf{4}$ demonstrated, $\mathrm{CO}_{2}$ laser energy was highly absorbed by the submucosal injection agents ${ }^{7}$.

\section{$\mathrm{CO}_{2}$ laser irradiation of resected porcine stomach}

The authors have previously reported on the effect of $\mathrm{CO}_{2}$ laser irradiation of resected porcine stomach tissue, thickness of the mucosal layer was $2 \mathrm{~mm}$, which was irradiated with a $\mathrm{CO}_{2}$ laser at an average power density of $1300 \mathrm{~W} / \mathrm{cm}^{2}$, and an irradiation time from 0 to10 $\mathrm{s}$ with or without submucosal injections. The result of this experiment was that, without submucosal injection, the mucosa was incised at $1 \mathrm{~s}$, the laser energy reached the muscle layer at $3 \mathrm{~s}$ and perforation through all layers occurred from $5 \mathrm{~s}$ onwards. Laser irradiation with submucosal injections resulted in incision of the mucosal layer only at $10 \mathrm{~s}$ and no damage to the muscle layer ${ }^{7)}$ (Figure 5). (a)

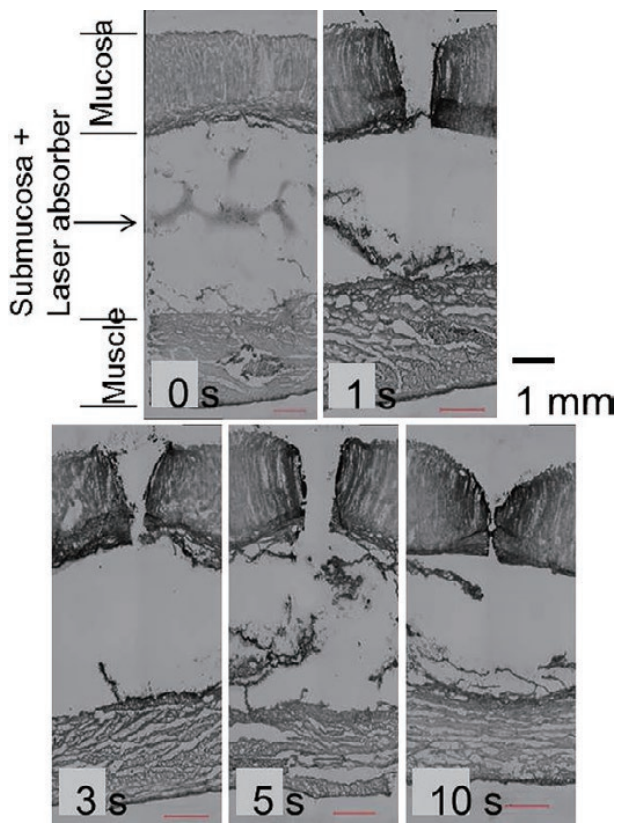

(b)
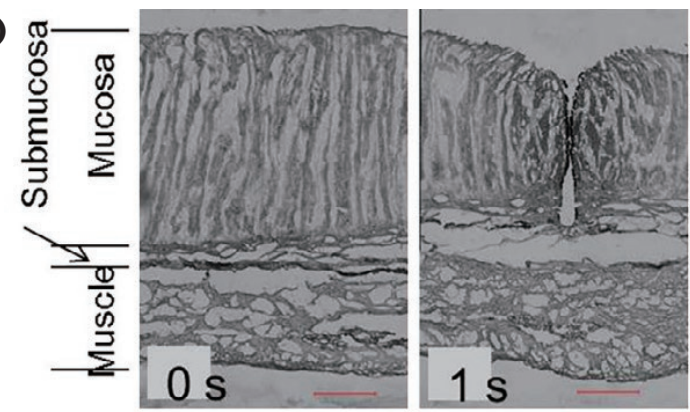

$1 \mathrm{~mm}$
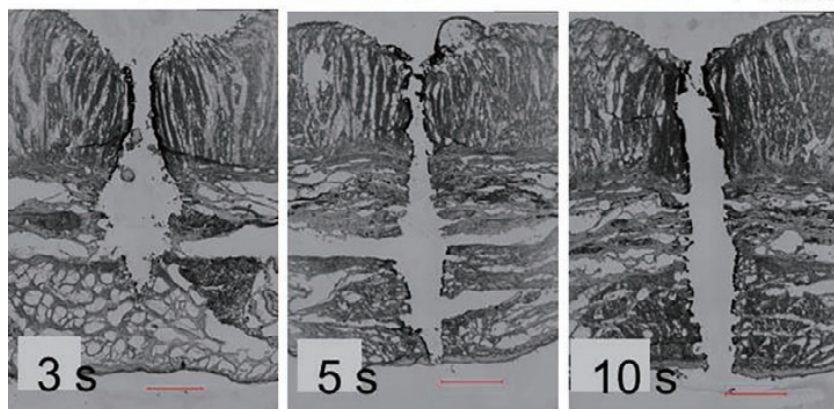

Fig. 5: The $\mathrm{CO}_{2}$ laser irradiation for porcine resected stomach with (a) or without (b) saline injection into the submucosal layer. Perforation was only observed without submucosal injection.

\section{CO2 laser}

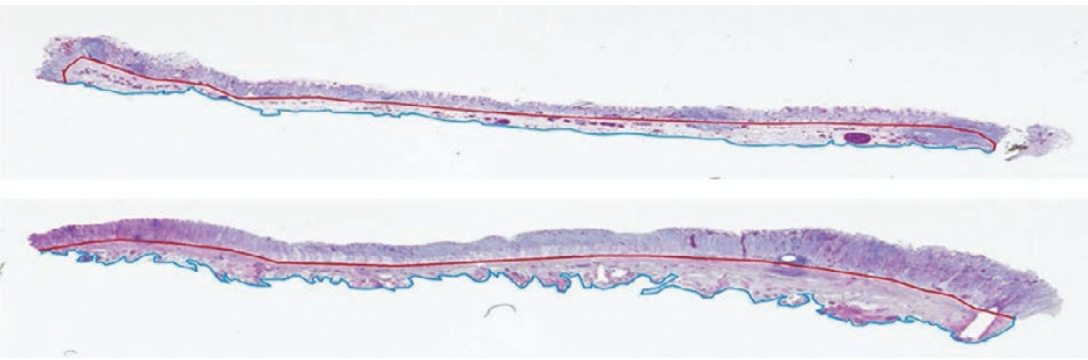

Fig. 6: Comparison of resected specimen between conventional and Laser ESD. Red line shows muscularis mucosa and blue line shows dissecting surface of submucosa. The smoothness of the dissecting surface was seen in the Laser ESD. 
ORIGINAL ARTICLES
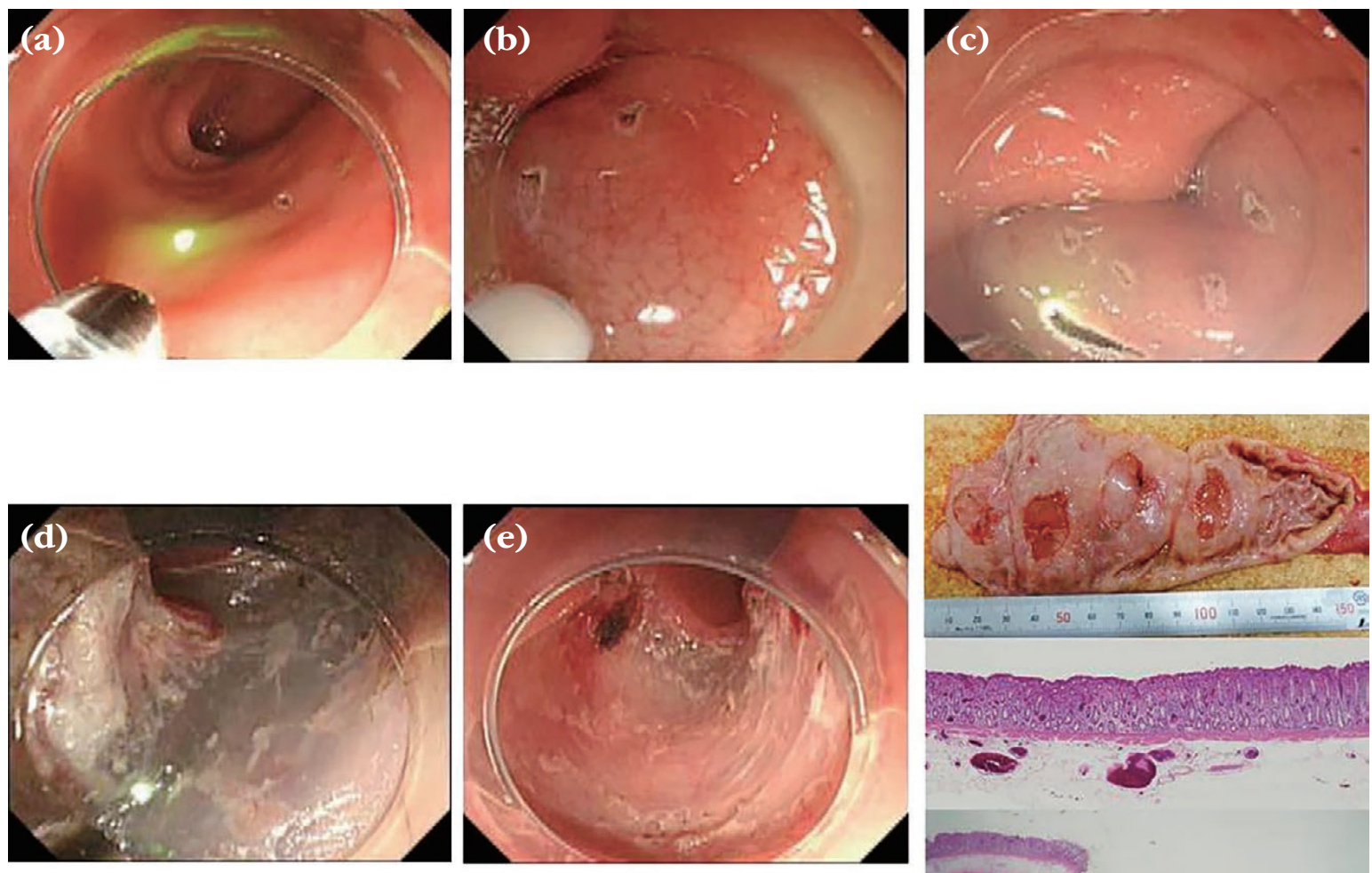

Fig. 7: Rectal Laser ESD for hypothetical lesion. (a) Marking. (b) Injection. (c) Mucosal incision. (d) Submucosal dissection. (e) Ulcer bed after ESD. (f) Resected specimen.
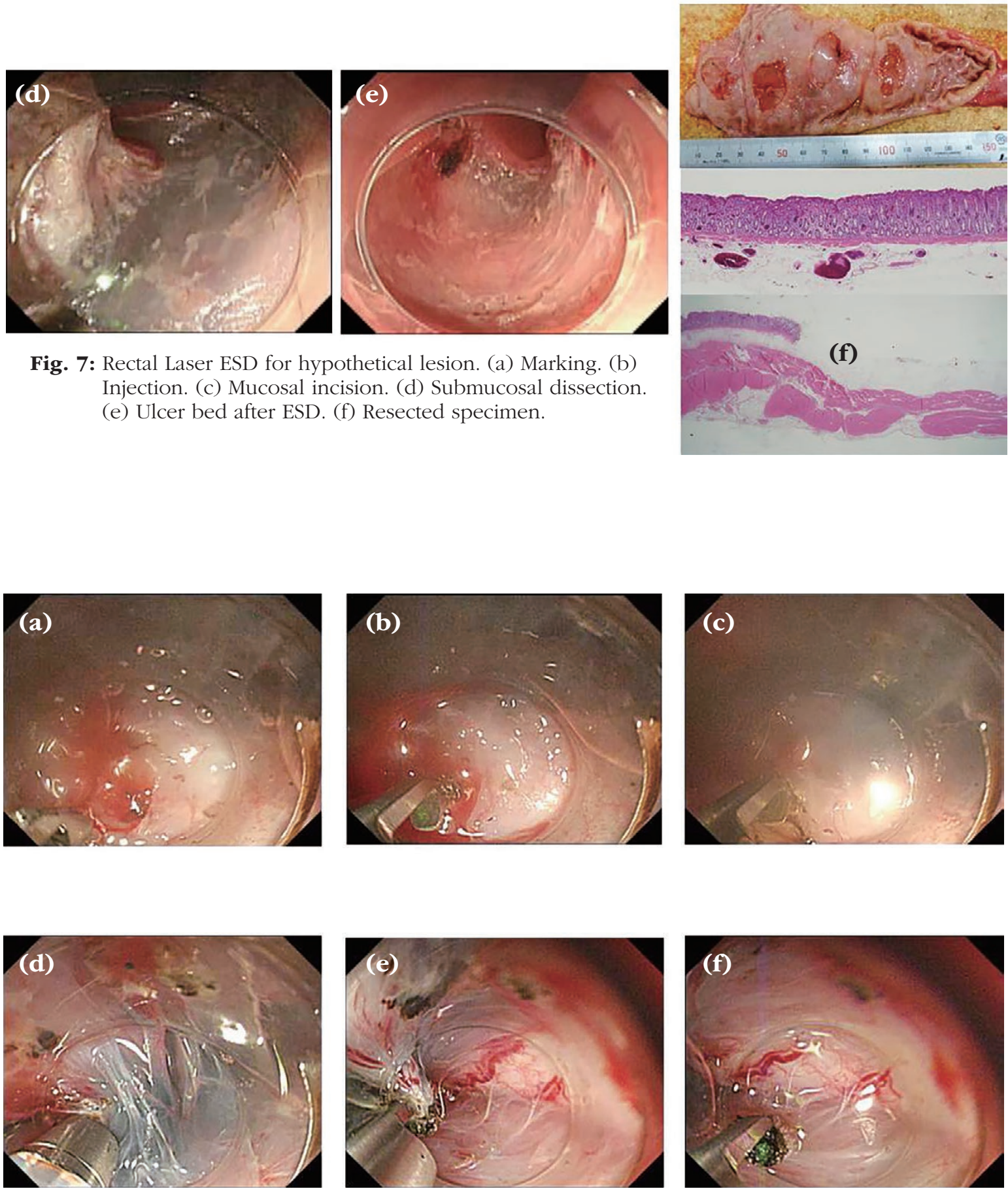

Fig. 8: "Hot tip" device for hemostasis and dissection. (a) Bleeding from vessel. (b) Coagulation by the tip of "Hot tip" device. (c) Hemostasis was seen. (d) Submucosal dissection by "Hot tip" device. (e) Vessel sealing by the blade of "Hot tip" device. (f) Submucosal dissection was finished successfully without damage of muscle layer. 


\section{In vivo experiments}

1. Based on these results, ESD was performed on the stomachs of minipigs (LWD, $18.5 \mathrm{~kg}$ ) under general anesthesia using both laser and conventional electrosurgical knife (Flushknife-BT 2.5 mm: FUJIFILM Corp., Japan), and the results regarding safety and efficacy were compared. Fourteen hypothetical lesions were marked with the laser on the anterior and posterior walls of the antrum, lower-, mid- and upper- body of the stomach. Each lesion measured $1 \mathrm{~cm}$ in diameter. The endoscope (GIF-Q260J: Olympus Corp., Japan) used was a widely accepted endoscope for ESD procedures. The submucosal injection agent was sodium hyaluronate (MucoUp: Seikagaku Corp., Japan) diluted with saline $1: 1$. The needle used for the injection was the impact flow $\mathrm{H}$ type 25 gauge needle (TOP Corp., Japan) and the endoscope tip attachment was the short ST hood (FUJIFILM Corp., Japan). The high frequency generator for the electrosurgical knife was the VIO200S (ERBE, Germany). The settings for the ESD in the mini pigs were the same as the ones used in human patients. Mucosal electrosurgical incisions were done at Endocut I mode (effect 2, duration 4, interval 2), while the submucosal dissections were performed with Forced coagulation mode (effect 3, $50 \mathrm{~W}$ ). The maximum output of the laser was $15 \mathrm{~W}$ but laser outputs of $1 \mathrm{~W}, 6-10 \mathrm{~W}$ and 3-5 W were used for marking, mucosal incision and submucosal dissection, respectively.

2. Similarly, laser ESD was performed on 6 hypothetical rectal lesions $2 \mathrm{~cm}$ in diameter, which had thinner walls, and the safety and efficacy were assessed. The laser outputs were $1 \mathrm{~W}$ for marking, $3 \mathrm{~W}$ for mucosal incisions and 3-5 W for submucosal dissections.

3. The hot-tip type hemostat was used experimentally to control hemorrhage during the procedures on the stomach. The hemostat was tested also for its capability in submucosal dissection. To ensure prompt response, the laser output was set at $10 \mathrm{~W}$.

These experiments were performed following approval by the ethics committee for animal experimentation within the Kobe Medical Device Development Center (MEDDEC).

\section{Results}

1. An expert in ESD, having performed over 2000 cases, and a novice with experience of less than 50 procedures each performed 7 ESD procedures on the mini pigs using both laser and the conventional electrosurgical knife. All resections were made en-bloc with no perforation. The average diameters of the resected specimens were $21.0 \pm 3.2 \mathrm{~mm}$ for the conventional electrosurgical knife ESD group and $24.3 \pm 8.0 \mathrm{~mm}$ for the laser ESD group, but the difference was not signifi- cant. The average procedure time was $9.4 \pm 6.6 \mathrm{~min}$ utes and $23.3 \pm 10.8$ minutes for the conventional electrosurgical knife ESD group and laser ESD group, respectively. Laser ESD took significantly longer $(p<$ 0.05 ), however, observation of the resected specimens revealed that the dissection surfaces were smoother and more uniform with less thermocoagulation in the laser ESD group compared to that of the electrosurgical knife ESD group (Figure 6).

2. Similarly, ESD was performed in the rectum with no perforation and the resected specimens showed uniform dissection surfaces with only mild thermocoagulation (Figure 7).

3. The novel hot-tip type hemostat achieved pinpoint coagulating hemostasis when using the tip and was also able to dissect the submucosal layer safely without causing perforation using the hooked part of the device (Figure 8).

\section{Discussion}

In this study the authors attempted to develop and introduce a novel, safer ESD procedure to the field of gastroenterology by applying the $\mathrm{CO}_{2}$ laser and utilizing the specific characteristics of mid-IR laser light. As stated above, the number of patients requiring ESD is growing around the world. In the case of thin-walled organ such as the colon, perforation caused by ESD is reported to be $5 \%$ in even the most experienced, specialized facilities in Japan. The perforation rate limited to Western countries rises to $8.6 \%$ and is one of the reasons that this procedure cannot be easily accepted. One of the reasons for this discrepancy of perforation rates is that endoscopists in Japan gained experience in ESD through gastric lesions where the wall of the stomach is relatively thick and gastric lesions are rather common in Japan. The endoscopists may subsequently step up to the more challenging ESD for colonic lesions. In Western countries, on the other hand, the gastroenterologists must face ESD of the colon outright since gastric lesions are scarce in these countries. The growing number of novel or newly developed devices to enhance the safety and efficiency of ESD reaching the market speaks for itself, and gives a strong indication that conventional ESD is reaching its limit.

In this study the authors proved that by replacing the electrosurgical knife with the $\mathrm{CO}_{2}$ laser and by injecting light absorbers such as saline into the submucosal layer, selective resection of the mucosa was possible while preventing perforation at the same time. Furthermore, during dissection of the submucosal layer, laser energy absorbed by water vaporized tissue slowly and dissections proceeded as if the tissue were melting which was completely different from dissection with electrosurgical knives, which was associated with sparking as the system made contact with tissue. In conventional ESD, 
the tip of the electrosurgical knife must be kept in contact with the tissue in an almost exquisitely precise fashion. Therefore, it has been reported that, with electrosurgical knife having needle-type tips, the risk of perforation increases. However, since laser ESD is performed in the non-contact method, the field of vision can be enlarged by drawing the scope back to visualize the whole procedural setting. Better visualization may allow easier maneuverability of the endoscope and may make ESD easier for even novice endoscopists.

Furthermore, the importance of ESD lies not only in the en-bloc resection of the lesions but also resection that can withstand detailed pathological evaluations. In resected specimen with excessive coagulation, the evaluation of tumor depth, venous and lymph duct invasion become difficult so acquiring a specimen with abundant submucosal tissue with a less coagulated layer is imperative. In this study, specimens from the laser ESD group were more uniform in thickness with less coagulated tissue.

Cautionary notes for laser ESD must be stated. One being the indication of laser ESD for lesions overlying ulcer scar where strong fibrosis of the submucosal layer may exist. The amount of light absorber injection may be insufficient, and the risk of perforation may increase. This would be the same for conventional ESD but since the coagulation depth of laser is less than that of electrosurgical knife, ESD with a lowered laser output may allow for a finer dissection and make it safer than conventional ESD. Adding blue dye such as indigo carmine to the light absorber may allow higher visualization of the submucosal layer and permit better judgement between submucosa and muscle layer. Another point to be aware of is the time taken for laser ESD. If the laser irradiation takes too long, the absorber may vaporize and therefore, perforation may become a risk and care must be taken.

The second cautionary note would be the lower hemostatic capability of laser ESD for hemorrhage during the procedure. Formerly, this was looked at as a tradeoff for the lower tissue damage of the laser compared to the electrosurgical knife, but was considered a major chal-

\section{References}

1: Patel CKN (1964): Continuous-wave laser action on vibrational rotational transitions of $\mathrm{CO}_{2}$, Phys. Rev., 136(A): A1187-A1193.

2: Sakita T, Koyama S, Ishii M et al. (1981): Early cancer of the stomach treated successfully with an endoscopic neodymium-YAG laser: report of a case, Am. J. Gastroenterol,76(5): 441-445.

3: Mimura S, Ito Y, Nagayo T, et al. (1996): Cooperative clinical trial of photodynamic therapy with photofrin II and excimer dye laser for early gastric cancer. Lasers Surg. Med., 19(2): 168-172.

4: Yano T, Muto M, Yoshimura K, et al. (2012): Phase I study of photodynamic therapy using talaporfin sodium and diode lenge. However, the development of the hot-tip type hemostat proved successful. Heating the tip of the hemostat with laser energy made possible the necessary blood vessel coagulation. Furthermore, the hooked part of the hemostat grasps tissue and can be used for dissection. Since laser is only transmitted between the hooks, it is thought that ESD for the afore mentioned lesions with severe submucosal fibrosis may allow safe dissection of these lesions without being the concern of perforation. The present hot-tip type of hemostat is still a prototype and further developments in its shape and rotational capabilities are warranted.

The last issue concerning laser ESD would be the potentially unsteady laser irradiation during mucosal incision. The non-contact mode of laser irradiation leads to instability of the point of incision compared with the electrosurgery contact mode, which could lead in turn to tremor-related imprecision of the laser beam. This was one of the reasons that laser ESD on the mini pigs took significantly more time than conventional ESD. We are presently developing a longer attachment to the tip of the endoscope. The longer attachment will allow the laser device to be fixed in the attachment and by pressing and fixing the tip of the attachment to the lesion, shake could be decreased, leading to more stable and efficient mucosal incision.

Endoscopic treatments using the $\mathrm{CO}_{2}$ laser promise many advantages over the conventional electrosurgical knife, but the laser must be used only after full understanding of its characteristics.

\section{Conclusion}

This study demonstrated that endoscopic treatments using the $\mathrm{CO}_{2}$ laser in combination with an injected light absorber was a safe and readily performable procedure. We are planning to apply for the approval of respective foreign and domestic agencies for the use of this medical technology on human patients and to make it commercially available. laser for local failure after chemoradiotherapy for esophageal cancer, Radiat. Oncol., 7: 113

5: Yano T, Kasai H, Horimatsu T, et al. (2017): A multicenter phase II study of salvage photodynamic therapy using talaporfin sodium (ME2906) and a diode laser (PNL6405EPG) for local failure after chemoradiotherapy or radiotherapy for esophageal cancer, Oncotarget, 8(13): 22135-22144.

6: Saito Y, Fukuzawa M, Matsuda T, et al. (2010): Clinical outcome of endoscopic submucosal dissection versus endoscopic mucosal resection of large colorectal tumors as determined by curative resection, Surg. Endosc., 24(2): 343-352.

7: Obata D, Morita Y, Kawaguchi R, et al. (2013): Endoscopic submucosal dissection using a carbon dioxide laser with 
submucosally injected laser absorber solution (porcine model), Surg. Endosc., 27(11): 4241-4249.

8: Fuccio L, Hassan C, Ponchon T, et al. (2017): Clinical out- comes after endoscopic submucosal dissection for colorectal neoplasia: a systematic review and meta-analysis, Gastrointest. Endosc., 86(1): 74-86.

\section{Acknowledgments}

This study was promoted by the "The development and overseas expansion of endoscopic laser treatment hardware based on domestic original technologies" project endorsed by the Japan Agency for Medical Research and Development. The authors would also like to show their greatest appreciation to Mr. Yoshihide Okagami, Mr. Akihito Hongoh, Mr. Katsumi Hiyoshi, Mr. Haruhiko Murakami and Mr. Yoshiteru Tamura from J. Morita MFG Corp. for their support and cooperation.

Editor's Note: This paper was originally published in Japanese in the Journal of Japan Society for Laser Surgery and Medicine, Vol. 38-4:413-420, 2018, and has been specially translated for inclusion in Laser Therapy as an English Original Article.

\section{Conflict of interest}

No conflict of interest exists. 